\title{
Low serum levels of vitamin D are associated with progression of subclinical atherosclerotic vascular disease in peritoneal dialysis patients: a prospective, multicenter study
}

\author{
Miguel Pérez Fontán ${ }^{\mathrm{a}, \mathrm{b}}$, Mercè Borràs Sans ${ }^{\mathrm{c}-\mathrm{e}}$, Maria Auxiliadora Bajo Rubio ${ }^{\mathrm{f}}$, Ana \\ Rodriguez-Carmona $^{\mathrm{a}}$, Angels Betriu ${ }^{\mathrm{d}, \mathrm{e}}$, José Maria Valdivielso ${ }^{\mathrm{d}, \mathrm{e}}$, Elvira Fernández ${ }^{\mathrm{c}-\mathrm{e}}$, \\ for the NEFRONA Study investigators
}

\author{
${ }^{a}$ Division of Nephrology, University Hospital A Coruña, and, \\ ${ }^{b}$ Group of Research on Cardiovascular and Metabolic Diseases, INIBIC, University of A Coruña, A Coruña, and, \\ ${ }^{c}$ Division of Nephrology, University Hospital Arnau de Vilanova, and, \\ ${ }^{d}$ UDETMA, University of Lleida, and, \\ ${ }^{e}$ IRB Lleida, Institut de Recerca Biomédica of Lleida, Lleida, and, \\ ${ }^{f}$ Division of Nephrology, University Hospital la Paz, Madrid, Spain
}

\begin{abstract}
Background: The prevalence of subclinical atherosclerosis and the main predictors of progression of this condition in patients undergoing peritoneal dialysis (PD) have been insufficiently investigated.

Objectives and method: Following a prospective, multicenter, observational design, we studied 237 patients who were treated with PD for $\geq 3$ months, without any clinical background of cardiovascular (CV) disease. Our objectives were the following: (1) to investigate the prevalence of subclinical atherosclerosis, as compared to a control group of age- and sex-matched healthy individuals, and (2) to disclose PD technique-related predictors of progression of disease during a 24-month follow-up period. We used vascular ultrasound for characterization of subclinical atherosclerotic disease.

Main results: A total of 123 patients (51.9\%) vs. 79 controls $(33.5 \%)$ presented $\geq 1$ carotid plaque, and 114 patients $(48.3 \%)$ vs. 72 controls $(30.5 \%) \geq 1$ femoral plaque, at baseline evaluation $(\mathrm{p}<0.0005)$. Progression of disease, either in clinical or ultrasound (new plaques) terms, affected $62.6 \%$ of patients. Multivariate analysis identified age, carotid intima-media thickness, presence of $\geq 1$ carotid plaque, and serum levels of $25 \mathrm{OH}$ vitamin $\mathrm{D}$ and C-reactive protein (CRP) at baseline as independent correlates of progression of atherosclerotic disease. On the contrary, PD techniquerelated variables did not show any association with this outcome.

Conclusions: Atherosclerotic vascular disease is frequent among asymptomatic patients undergoing PD. Older age, pre-existent disease (assessed by vascular ultrasound), and serum levels of $25 \mathrm{OH}$ vitamin D and CRP are independent markers of the progression of this condition. These findings may contribute to improve identification of subpopulations with a high risk of $\mathrm{CV}$ events, deserving intensified measures of prevention.
\end{abstract}

\section{Keywords:}

Carotid atherosclerosis; Peritoneal dialysis; Vitamin D 


\section{Introduction}

Cardiovascular (CV) disease (CVD) represents the main cause of mortality among patients with endstage renal disease (ESRD). This disorder has a complex pathogenesis and results from the interaction of many traditional and non-traditional CV risk factors, which are very prevalent, in these patients. These factors are related to preexistent comorbidities, adverse consequences of the chronic kidney disease (CKD) environment, and undesired effects of renal replacement therapies (RRTs) [1].

Atherosclerosis is a primary contributor to CVD in patients with ESRD [1]. A significant proportion of affected individuals have a background of atherothrombotic events before RRT is initiated, and many others will undergo fatal or nonfatal events of this type during follow-up. Consequently, prevention of the progression of atherosclerotic disease is an essential part of the management of patients on dialysis. Subjects with a back-ground of previous CV events, and also those who ac-cumulate a high burden of CV risk factors, represent obvious targets for intensified preventive measures. Detection of subclinical atherosclerosis may help to re-fine identification of individuals with an increased CV risk. Highresolution vascular ultrasonography (vUS) is a reliable and relatively simple tool for the study of arteriosclerotic vascular disease. Increased carotid intima-media thickness (cIMT) and the presence of calcified and non-calcified plaques are strong predictors of CV events in the general population [2-5], and have been claimed to portend similar outcomes in patients with ESRD [6-8].

The impact of the different modalities of dialysis on the progression of atherosclerotic vascular disease is a matter of controversy. In particular, peritoneal dialysis (PD) associates some pro-atherogenic features [9], which may compromise the long-term CV outcome of affected patients [10]. vUS could contribute to characterize the atherosclerotic burden of PD patients, but available in-formation is based on cross-sectional comparisons with hemodialysis patients, with inconclusive results [11-13]. A longitudinal approach could be more effective to de-fine the main risk factors for progression of atherosclerotic vascular disease in this population but, to our knowledge, this approach has not been undertaken so far.

We present the results of a prospective, multicenter, observational study, oriented to (1) investigate the prevalence of subclinical atherosclerotic vascular disease in asymptomatic patients undergoing chronic PD, and (2) identify clinical correlates of progression of this disorder, with a particular focus on PD technique-related factors, in these patients.

\section{Method}

\section{General study design and participants}

The NEFRONA project is a Spanish multicenter, observational, prospective study, designed to investigate the atherosclerotic bur-den of patients in different stages of CKD, including relatively large samples of patients with ESRD treated with dialysis. A large control group of individuals without CKD is also available. The general design and objectives of the project have been reported in detail elsewhere [14-16]. The study was able to recruit 2,445 CKD patients, 18-75 years of age, from 81 different Spanish hospitals, between October 2010 and June 2012. Remarkably, NEFRONA aimed to investigate only patients without overt atherosclerotic disease at the start of follow-up. Consequently, candidates with a background of previous coronary, cerebrovascular, peripheral vascular, or any other type of CV event were not eligible.

For the present study, we investigated all the patients treated with PD who were recruited for NEFRONA. The study population comprised 237 patients prevalent on PD (previous follow-up $\geq 3$ months under this modality of RRT) from 37 different units, distributed all over Spain. A control group, composed of 237 age- and sex-matched subjects without CKD, was built for comparisons. The study had a 2 -fold objective. First, we aimed to investigate the prevalence and extension of subclinical atherosclerotic disease in PD patients as compared with healthy controls. For this purpose, a thorough base-line evaluation was performed. Second, we focused our analysis on disclosing markers of progression of subclinical disease in PD patients, for which a follow-up visit was scheduled 24 months after the initial evaluation. Patients $(n=8)$ who had significant $(>70 \%)$ stenotic carotid plaques $(n=4$; vUS) or an ankle-brachial index (ABI) $<0.7(n=6)$ at baseline evaluation ( vide infra) were considered to present clinically significant vascular disease, and did not undergo further analysis. We also excluded 
from longitudinal analysis those patients who received a renal allograft, who were lost to follow-up, or who died for non-CV reasons within 24 months after the baseline visit. Subjects who suffered a CV event during the aforementioned period were considered for longitudinal analysis (see below), but did not undergo the scheduled 24-month visit (vUS).

The study complied with the principles of the Declaration of Helsinki. The protocol was approved by the Institutional Commit-tee of each participating center, and written informed consent was obtained from all participants.

\section{Collection and management of clinical and biochemical data}

At the time of recruitment, we gathered information about cur-rent health status, medical history, preexistent $\mathrm{CV}$ risk factors, and drug therapies. A general physical examination was performed, and $\mathrm{ABI}$ was estimated, as previously described [17]. Blood samples were retrieved for biochemical parameters (Table 1) as close as possible to the baseline visit, and always within 3 months of the baseline vUS study. Biochemical determinations were performed, for the most part, in the participating centers, with the help of autoanalyzers. Parathyroid hormone levels were standardized using a conventional conversion method [18] to compensate for inter-method variability between different centers. Determinations of highsensitivity C-reactive protein (CRP; Immunoturbidimetry, Roche Hitachi, Modular Analytics, Indianapolis, IN, USA) and 25-hydroxy-vitamin D (ELISA, IDS, Tyne and Wear, UK) levels were carried out in a central laboratory to avoid variability among methods. Residual kidney function glomerular filtration rate (GFR) was estimated as the mean of urea and creatinine renal clearances.

vUS explorations were performed by 3 itinerant teams, who also performed anthropometric and ABI estimations, and retrieved blood samples.

Table 1. Main baseline characteristics of the study population and control group

\begin{tabular}{|c|c|c|c|}
\hline & PD patients & Controls & $p$ value \\
\hline & 237 & 237 & \\
\hline Age, years & $51.2(13.7)$ & $51.4(12.9)$ & 0.87 \\
\hline Gender, male/female, \% & $58.1 / 41.9$ & $58.1 / 41.9$ & 1.00 \\
\hline Diabetes mellitus, \% & $43(18.1)$ & $35(14.8)$ & 0.38 \\
\hline Tobacco (nonsmoker/former/smoker), \% & $43.2 / 37.7 / 19.1$ & $44.5 / 38.6 / 16.9$ & 0.84 \\
\hline Pulse pressure, $\mathrm{mm} \mathrm{Hg}$ & $58.4(17.9)$ & $52.0(12.1)$ & 0.0005 \\
\hline Body mass index, $\mathrm{kg} / \mathrm{m}^{2}$ & $26.7(4.9)$ & $28.1(4.6)$ & 0.001 \\
\hline Total cholesterol, mg/dL & $180.1(42.8)$ & $195.7(35.0)$ & 0.005 \\
\hline HDL cholesterol, mg/dL & $49.4(14.9)$ & $52.8(14.9)$ & 0.02 \\
\hline LDL cholesterol, mg/dL & $103.6(34.1)$ & $120.1(31.4)$ & 0.0005 \\
\hline Triglycerides, mg/dL & $123(35-419)$ & $94(31-662)$ & 0.001 \\
\hline Serum phosphate, $\mathrm{mg} / \mathrm{dL}$ & $5.1(1.2)$ & $3.5(0.6)$ & 0.0005 \\
\hline Serum $25 \mathrm{OH}$ vitamin $\mathrm{D}, \mathrm{ng} / \mathrm{mL}$ & $12.8(5.5)$ & $20.6(8.4)$ & 0.0005 \\
\hline Serum $1,25 \mathrm{OH}$ vitamin $\mathrm{D}, \mathrm{pg} / \mathrm{mL}$ & $7.7(4.8)$ & $33.0(14.4)$ & 0.0005 \\
\hline Uric acid, $\mathrm{mg} / \mathrm{dL}$ & $6.0(1.2)$ & $5.1(1.4)$ & 0.0005 \\
\hline Parathyroid hormone, $\mathrm{pg} / \mathrm{mL}^{*}$ & $277.5(214.2)$ & - & - \\
\hline
\end{tabular}

Figures denote mean values (SD) or $n(\%)$. Triglycerides and C-reactive protein presented as median values (range).

* Normalized for assay. 


\section{Vascular Ultrasound}

B-mode ultrasound of the carotid and femoral arteries was per-formed using the Vivid BT09 device (General Electric Instruments, Chicago, IL, USA), with the help of 6-13 MHz broadband linear array probes. The measurement of IMT and the search for atheromatous plaques was performed by a unique reader in a blinded fashion, using the semi-automatic software EchoPAC Dimension (General Electric Healthcare, Chicago, IL, USA). We previously assessed the quality of the reading and the intraobserver variability, using a sample of 20 individuals in whom estimations were per-formed 3-5 times at different days. A kappa coefficient of 1 was obtained, indicating optimal intraobserver reliability.

Bilateral US imaging was performed with the subjects in a supine position. For carotid US, the head was turned $45^{\circ}$ contra-lateral to the side of the probe, and cIMT was measured in the last centimeter of the far wall of the common carotid artery, the bifurcation section, and finally, the first centimeter of the internal carotid artery. Measurements were made in plaque-free arterial segments. The presence of atheromatous plaques in each of the mentioned points was defined by an IMT $\geq 1.5 \mathrm{~mm}$ protruding to the lumen, following the recommendations of the ASE Consensus Statement [18] and the Mannheim cIMT Consensus report [19].

\section{Study Variables}

For cross-sectional comparisons between PD patients and healthy controls, the main study variables were cIMT and the presence of atheromatous plaques in the carotid and femoral territories. For the latter purpose, we created plaque scores, which resulted from the addition of the number of points scrutinized ( 6 for the carotid territories, including common, bifurcation, and internal carotid arteries on each side, and 4 for the femoral territories, including common and superficial femoral arteries on each side) in which at least 1 plaque was detected. Thus, the score ranged between 0 (no plaques) and 6 (all sites examined with plaque) in the carotid territories, and between 0 and 4 in the fem-oral territories.

For the longitudinal study, the main outcome variable was progression of atherosclerotic disease, arbitrarily defined as 1 or 2 of the following: appearance of, at least, a new carotid plaque in the 24-month visit (vUS), or significant (demanding in-center evaluation or management) CV event during the 24-h follow-up period. Only carotid, but not femoral plaques were used to build this score, to facilitate comparability with other studies based on vUS. The study had an exploratory design, meaning that we considered as study variables all those collected at baseline. However, we focused on some with a specific interest in PD patients, including time on dialysis, blood pressure levels, GFR, peritoneal transport characteristics, conditions of prescription, lipid profiles, serum vitamin D levels, plasma albumin levels, and bone mineral disease markers. Peritoneal transport was characterized from the D/P quotient of creatinine at 240' at the time of initiation of the study, according to the local standards of each participating center.

\section{Data Analysis}

Numeric variables are presented as mean values with SD, except those with a markedly abnormal distribution (median with range). Categorized variables are presented as absolute numbers (\%). Main univariate comparisons were produced by means of Student $t$ test, ANOVA, Mann-Whitney test, and $\chi^{2}$ distribution. The Spearman's correlation coefficient was used to analyze correlations among numerical variables. Statistical significance was de-fined by a $p$ value $<0.05$.

We used stepwise logistic regression analysis to investigate factors associated with the progression of atherosclerotic disease. As previously stated, all the recorded baseline variables were considered for analysis. Only variables with a univariate association to progression with a $p$ value $<0.10$, as also those with a specific interest in PD patients (vide supra) were explored during multivariate analysis. Furthermore, we constructed 2 different multivariate approaches, first focusing on clinical and laboratory variables (Model 1), and then introducing objective baseline estimations of vascular disease (baseline cIMT, baseline number of plaques, pulse pressure, and ABI; Model 2). Only first-degree interaction terms were scrutinized.

The SPSS 19.0 software was used for data analysis. 


\section{Results}

\section{Baseline Data}

The essential baseline characteristics of the study group and matched controls are presented in Table 1. With the notable exceptions of total and LDL cholesterol levels, the CV risk profile was more unfavorable in PD patients than in controls. The essential conditions of prescription for patients are displayed in Table 2 .

Table 2. Main baseline dialysis- and prescription-related variables in PD patients

\begin{tabular}{lc}
\hline & \\
Time on dialysis, months & $19.9(13.7 ;$ range 3-90) \\
Category of small solute transport (S/SA/FA/F), \% & $9.4 / 26.5 / 52.6 / 11.5$ \\
Modality of PD (CAPD/automated PD), \% & $43.6 / 56.4$ \\
Volume of dialysate, mL/24 h & $9,232(8,504)$ \\
Type of PD solution (high/low in GDP), \% & $62 / 175(26.2 / 73.8)$ \\
Icodextrin for long dwell, \% & $116(49.2)$ \\
Residual kidney function (GFR), mL/min & $5.8(5.2)$ \\
Diuresis, mL/24 h & $1,251(1,200)$ \\
Peritoneal, Kt/V & $1.48(1.44)$ \\
Total, Kt/V & $2.52(2.43)$ \\
Daily ultrafiltration, mL/24 h & $759(714)$ \\
Treatment with antihypertensives, \% & $214(90.3)$ \\
Treatment with RAAS antagonists, \% & $148(62.4)$ \\
Treatment with statins, $\%$ & $137(57.8)$ \\
Treatment with antiplatelet drugs, $\%$ & $54(22.8)$ \\
Treatment with vitamin D or analogues (any type), \% & $120(50.6)$ \\
& \\
\hline
\end{tabular}

Figures denote mean values (SD) or $n(\%)$

S, slow; SA, slow average; FA, fast average; F, fast; GDP, glucose degradation products; RAAS, renin-angiotensin-aldosterone system.

PD patients presented a different ABI pattern, when compared with controls (Table 3). Both abnormally low and high values were more frequent in the former group. As refers to baseline arterial US findings, mean cIMT was similar in patients and controls (Table 3). On the contrary, both carotid and femoral plaques were significantly more prevalent in PD patients (Table 3).

Table 3. Baseline markers of atherosclerotic vascular disease

\begin{tabular}{|c|c|c|c|}
\hline & Patients & Controls & $p$ value \\
\hline Ankle-brachial index & $1.07(0.25)$ & $1.03(0.10)$ & 0.052 \\
\hline Distribution & & & 0.0005 \\
\hline $0.70-0.90$ & $27(11.4)$ & $20(8.4)$ & \\
\hline $0.91-1.40$ & $194(81.9)$ & $217(91.6)$ & \\
\hline$>1.40$ & $10(4.2)$ & $0(0)$ & \\
\hline Number of patients with at least one carotid plaque, $\%$ & $123(51.9)$ & $79(33.5)$ & 0.0005 \\
\hline Plaque score for carotid territories & $1.16(1.46)$ & $0.64(1.08)$ & 0.0005 \\
\hline Number of patients with at least one femoral plaque, $\%$ & $114(48.3)$ & $72(30.5)$ & 0.0005 \\
\hline Plaque score for femoral territories & $0.93(1.17)$ & $0.50(0.86)$ & 0.0005 \\
\hline
\end{tabular}

Figures denote mean values (SD) or $n(\%)$. 
Among patients, the baseline carotid plaque score correlated with age $(r=0.50, p<0.0005$, Spearman), diabetes $(1.9 \pm 1.5$ vs. $1.0 \pm 1.4$ in non-diabetics, $p<0.0005)$, pulse pressure $(r=0.18, p=$ $0.017)$, body mass index $(r=0.15, p=0.018)$, plasma albumin $(r=-0.21, p=0.001)$, and se-rum levels of $25 \mathrm{OH}$ vitamin $\mathrm{D}(r=-0.17, p=0.009)$ and CRP $(r=0.18, p=0.006$; all other variables presented in Tables 1 and 2 ; not significant).

\section{Follow-Up}

Twenty-eight PD patients (11.8\%) suffered at least $1 \mathrm{CV}$ event, and 12 of them died due to CV reasons, during the 24-month follow-up study period. These patients were considered for analysis of vascular disease progression, but not of plaque progression (vUS). According to the study protocol, we excluded 8 patients $(3.4 \%)$ from longitudinal analysis who died of non-CV reasons, 5 patients $(2.1 \%)$ who were lost to follow-up, and 119 patients (50.2\%) who received a kidney allograft during the 24month follow-up period. Overall, 99 patients were available for the analysis of predictors of disease progression, including the 28 who suffered at least $1 \mathrm{CV}$ event during follow-up, and 71 more individuals who completed the 24-month vUS assessment.

As compared with patients included in the follow-up analysis, excluded patients were younger $(48.9 \pm$ 12.8 vs. $53.8 \pm 14.1$ years, $p=0.005)$, presented lower serum levels of CRP $(0.16 \mathrm{mg} / \mathrm{dL}$ [median range 0.02-5.29] vs. $0.33 \mathrm{mg} / \mathrm{dL}$ [0.02-13.17, $p=0.001$, Mann-Whitney] and had marginally lower values of pulse pressure [56.2 \pm 16.9 vs. $60.7 \pm 18.7, p=0.056$ ] and number of plaques [carotid and femoral] at baseline [1.8 \pm 2.3 vs. $2.4 \pm 2.2, p=0.078]$; all other variables quoted in Tables 1 and 2 not significant).

When only subjects with baseline and 24-month vUS evaluation were considered $(n=71), 12$ patients $(17.4 \%)$ had an increase of $>0.1 \mathrm{~mm}$ in the mean cIMT between both assessments. Moreover, 34 patients $(47.9 \%)$ presented one $(n=24,33,8 \%)$ or more $(n=10,14.1 \%)$ new carotid plaques, and 37 patients $(52.1 \%)$ presented one $(n=25,35.1 \%)$ or more $(n=12,16.9 \%)$ new femoral plaques. No patient appeared to develop severely stenotic $(>70 \%)$ carotid plaques on follow-up vUS.

Table 4 shows the distribution of baseline variables, according to disease progression during followup. The multivariate, clinical model for baseline predictors of dis-ease progression (Table 5, Model 1) identified older age, lower serum levels of $25 \mathrm{OH}$ vitamin $\mathrm{D}$, and higher serum levels of CRP as independent correlates of this outcome. The inclusion of objective estimations of baseline vascular disease resulted in 2 different predictive models (Table 5). Model 2a included carotid plaques at baseline as the most consistent predictor of disease progression, while serum levels of $25 \mathrm{OH}$ vitamin D and CRP persisted as independent predictors of disease progression. Model 2b, which was slightly less accurate, identified baseline mean cIMT as an alternative, consistent predictor of the main outcome. We did not detect any significant interaction term between variables in either model. While the association between CRP levels and progression of disease was largely restricted to patients in the higher tertile for this factor, the association of $25 \mathrm{OH}$ vitamin to $\mathrm{CV}$ disease progression was essentially linear, without apparent cutoff points. Remarkably, neither diabetes nor treatment with statins, renin-angiotensin-aldosterone system (RAAS) antagonists nor vitamin D (any type) were identified as independent markers of disease progression after controlling for age, baseline carotid plaque score, or baseline cIMT 
Table 4. Correlation between main baseline variables and atherosclerotic disease progression: univariate analysis

\begin{tabular}{|c|c|c|c|}
\hline & Progession $(n=62)$ & No progession $(n=37)$ & $p$ value \\
\hline Age, years & $57.1 \pm 11.8$ & $48.4 \pm 14.7$ & 0.002 \\
\hline Gender, male/female, $\%$ & $64.5 / 35.5$ & $54.1 / 45.9$ & 0.35 \\
\hline Diabetes mellitus, \% & 27.4 & 8.1 & 0.021 \\
\hline Tobacco (non-smoker/former/smoker), $\%$ & $37.1 / 43.5 / 19.4$ & $40.5 / 43.2 / 16.2$ & 0.90 \\
\hline Systolic blood pressure, $\mathrm{mm} \mathrm{Hg}$ & $149.1 \pm 23.5$ & $143.7 \pm 23.7$ & 0.28 \\
\hline Body mass index, $\mathrm{kg} / \mathrm{m}^{2}$ & $26.7 \pm 4.4$ & $26.7 \pm 6.2$ & 0.96 \\
\hline Total cholesterol, mg/dL & $187.2 \pm 52.4$ & $183.5 \pm 40.3$ & 0.67 \\
\hline HDL cholesterol, mg/dL & $48.2 \pm 15.5$ & $51.8 \pm 16.2$ & 0.28 \\
\hline LDL cholesterol, mg/dL & $108.0 \pm 40.7$ & $106.2 \pm 27.5$ & 0.82 \\
\hline Triglycerides, mg/dL & $134(50-376)$ & $121(35-250)$ & 0.85 \\
\hline Hemoglobin, g/dL & $12.0 \pm 1.6$ & $12.1 \pm 1.4$ & 0.80 \\
\hline Serum $25 \mathrm{OH}$ vitamin $\mathrm{D}, \mathrm{ng} / \mathrm{mL}$ & $11.9 \pm 5.6$ & $14.6 \pm 5.0$ & 0.019 \\
\hline Serum $1,25 \mathrm{OH}$ vitamin $\mathrm{D}, \mathrm{pg} / \mathrm{mL}$ & $7.8 \pm 3.7$ & $7.2 \pm 2.8$ & 0.37 \\
\hline Uric acid, mg/dL & $6.2 \pm 1.2$ & $5.6 \pm 1.0$ & 0.015 \\
\hline Parathyroid hormone, $\mathrm{pg} / \mathrm{mL}$ & $303.6 \pm 312.7$ & $258.2 \pm 182.0$ & 0.42 \\
\hline Ankle-brachial index & $1.13 \pm 0.35$ & $1.10 \pm 0.25$ & 0.59 \\
\hline Baseline cIMT, mm & $0.70 \pm 0.13$ & $0.60 \pm 0.11$ & 0.0005 \\
\hline Baseline carotid plaque score & $1.71 \pm 1.33$ & $0.57 \pm 1.11$ & 0.0005 \\
\hline Baseline femoral plaque score & $1.11 \pm 1.13$ & $0.69 \pm 1.11$ & 0.033 \\
\hline GFR, mL/min & $5.9 \pm 5.8$ & $6.2 \pm 4.2$ & 0.80 \\
\hline $\mathrm{Kt} / \mathrm{V}$ & $2.58 \pm 0.69$ & $2.36 \pm 0.53$ & 0.09 \\
\hline Volume of dialysate, $\mathrm{mL} / 24 \mathrm{~h}$ & $9,399 \pm 4,109$ & $8,597 \pm 4,112$ & 0.47 \\
\hline
\end{tabular}

cIMT, carotid intima-media thickness; GDP, glucose degradation products; RAAS, renin-angiotensin-aldosterone system. Numerical variables presented as mean (SD), except triglycerides and C-reactive protein (median with range). Categorized variables presented as \%. 


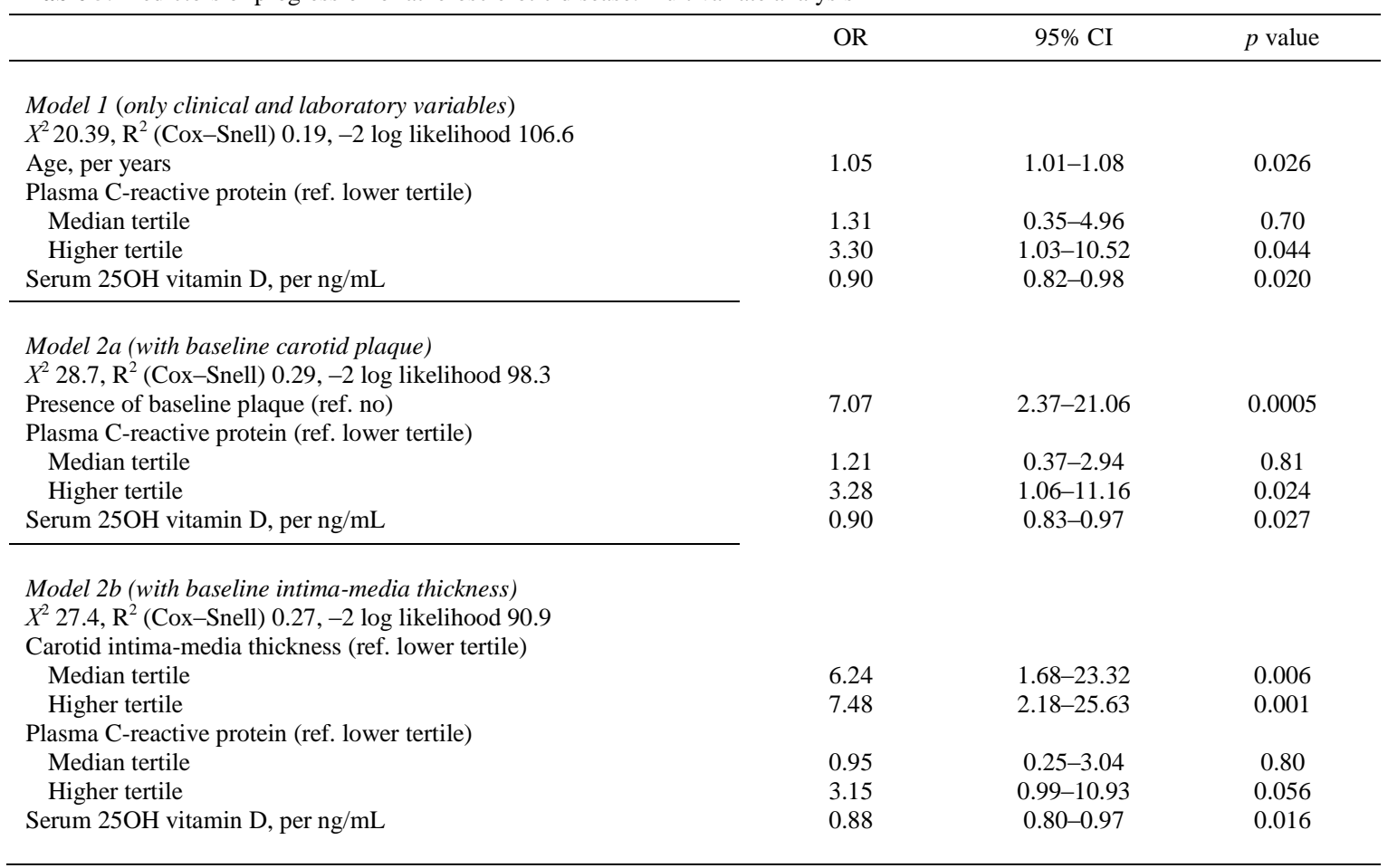

Stepwise logistic regression analysis. Outcome variable: progression of atherosclerosis during follow-up (either appearance of at least one new carotid plaque or cardiovascular event during the 24-month follow-up period). All other variables (clinical, laboratory, treatment-related or PD-related) not significant.

\section{Discussion}

The results of our study show that atherosclerotic disease is very prevalent in asymptomatic patients treated with PD. Moreover, the disease is progressive in a high proportion of patients, even after a relatively short follow-up of 24 months. The proportion of patients suffering CV events $(11.8 \%)$ or developing new carotid (47.9\%) or fem-oral (52.1\%) plaques during the 24-month follow-up was remarkable, considering that only patients without overt $\mathrm{CV}$ disease at baseline were included in the analysis. Similar findings have been reported for patients with CKD [20] and unselected populations on chronic dialysis [21]. These observations are worrisome, but not totally unexpected, given the high burden of traditional and non-traditional CV risk factors supported by these patients, resulting in elevated rates of $\mathrm{CV}$ mortality [1]. Patients undergoing PD are not an exception to this rule [9]. In fact, some factors specifically associated to this technique, including volume overload, peritoneal loading with dialysis solutions rich in glucose and glucose degradation products, and a continuous peritoneal leak of proteins and other macromolecules, may contribute to the well-known late surge in the CV risk of these patients [10].

$\mathrm{CV}$ events represent the most direct way to assess progression of CV disease. However, detecting this evolution before these events occur may permit a more accurate and profitable stratification of risk, helping to define subsets who could be a subject of intensified prevention measures. This strategy demands both tight monitoring and management of modifiable risk factors and a systematic use of reliable estimators of asymptomatic CV disease, including echocardiography and vUS. Regarding the latter, cIMT and the presence of carotid plaques are well standardized tools [18, 19], and have demonstrated to be highly predictive of CV risk, both in the general population [2-5] and in the particular case of patients with ESRD [7, 8, 21].

Comparing the significance of different markers of progression of subclinical CV disease in patients with ESRD treated with PD is a difficult task, due to the large set of factors with a potential effect on this outcome [9]. In our study, age and the presence of arterial disease (either increased cIMT or presence of plaques) at baseline were most consistent predictors of the risk of disease progression. A high 
inflammatory score (as estimated from CRP) also portended a significant risk of disease progression, in agreement with previous studies [22]. On the other hand, baseline serum levels of $25 \mathrm{OH}$ vitamin $\mathrm{D}$, but not $1,25 \mathrm{OH}$ vitamin $\mathrm{D}$, kept an independent, significant association with the risk of progression of CV dis-ease. The consistency of this association was remarkable and agrees with previous studies, showing that low se-rum vitamin D levels portend mortality, and more specifically CV outcomes, both in the general population [23, 24] and in patients with different stages of CKD [7, 20, 25-28], including those treated with PD $[29,30]$. However, the significance of this association still needs some clarification. For instance, it is unclear if the association is linear or if, alternatively, CV risk is restricted to patients with very low levels of this hormone $[25,27,28]$. The capacity of treatment with native vitamin $\mathrm{D}$ or its derivatives to improve $\mathrm{CV}$ outcomes of vitamin D-deficient patients is another matter of controversy [24, 28, 31, 32-37].

The mechanisms by which vitamin D is protective for the CV system have been a subject of great interest in the last years, and represent a good example of the pleiotropic effects of this hormone. First, vitamin D may preserve endothelial function by different mechanisms, which include direct stimulation of NO synthetase, modulation of angiogenesis, downregulation of inflammatory damage, protection against oxidative stress, and regulation of endothelium-derived contracting factors [23]. Vitamin D may also exert beneficial effects at the level of the vessel wall, including regulation of the proliferation, migration and stability of vascular smooth muscle cells, and modulation of fibrosis. Local regulation of $\mathrm{T}$ lymphocytes and macrophage function at the level of the vessel wall may represent another mechanism of vascular protection by vitamin D [38]. On the contrary, the evidence regarding the role of this factor in the regulation of vascular calcification is more controversial [23] although in the particular case of patients with CKD, there are some data linking vitamin D deficiency with progression of coronary calcification [25]. On the other hand, vitamin D may also exert some indirect effects on the genesis and progression of atherosclerosis. For instance, low levels of this hormone have been associated with defective insulin secretion, insulin resistance, dyslipidemia, and overactivity of the RAAS system [23]. All the potential consequences of vitamin D deficiency may be particularly relevant in PD patients, who present very low levels of this vitamin, not only when compared with normal individuals, but also with patients on earlier stages of CKD or those treated with hemodialysis [30].

Some negative findings in our study may deserve comment. For instance, diabetes was a significant correlating factor of atherosclerotic disease at baseline, and portended progression of the condition on univariate (Table 4), but not on multivariate analysis, which could be unexpected. The explanation for this circumstance is unclear, but the limited statistical power of the study may have obscured the role of this factor. On the other hand, most PD-related variables did not show any association with progression, suggesting that these factors may not be very influential, at the time of predicting patients' outcomes. We did not record data on peritoneal protein leak for this study. Peritoneal protein excretion has been linked to general and CV mortality in PD patients $[39,40]$. The potential mechanisms underlying this association not only include dyslipidemia and the generation of a prothrombotic environment, but also the performance of this factor as a surrogate of endothelial dysfunction. Interestingly, global peritoneal protein excretion keeps a close correlation with the peritoneal leak of vitamin D-binding protein, although several studies have challenged the influence of this factor on serum levels of vitamin D [41, 42]. Finally, treatment with statins, RAAS antagonists, or vitamin D did not show an association with disease progression. These findings are not unexpected, due to the significant risk of prescription bias, under the design of this study. A randomized trial design should be necessary to clarify this question. Treatment with statins has been shown to exert a marginal beneficial effect on CV risk in PD patients [43].

This study has significant limitations. Despite a multicenter design, the number of patients recruited may have been insufficient, given the large number of covariables considered. The high number of PD dropouts before completing the protocol contributed to this limitation, and may also have introduced a selection bias, because a majority of dropouts were due to kidney transplantation. Among the strengths of the study, we should mention its longitudinal, prospective, multicenter design, and the quality of data, including well designed vUS explorations and a centralized estimation of variables which came out to represent main findings of the investigation, including serum levels of vitamin D.

In summary, atherosclerotic vascular disease is very prevalent among asymptomatic patients undergoing chronic PD. Moreover, the disease progresses in a high proportion of these patients during follow-up. Older age, mean cIMT, or the detection of at least one carotid plaque during baseline vascular US screening, and high serum levels of CRP are independent predictors of disease progression. Importantly, lower serum levels of $25 \mathrm{OH}$ vitamin $\mathrm{D}$ consistently portend this outcome. These findings 
may contribute toward improving the identification of subpopulations with a high risk of CV events and mortality, deserving intensified measures of prevention.

\section{Statement of Ethics}

This study was approved by the ethical committees of all the participating centers, and written informed consent was obtained from all participants.

\section{Disclosure Statement}

The authors have no conflicts of interest to declare.

\section{References}

1. Wanner C, Amann K, Shoji T: The heart and vascular system in dialysis. Lancet 2016; 388: 276-284.

2. O’Leary DH, Polak JF, Kronmal RA, Manolio TA, Burke Gl, Wolfson SK Jr: Carotid-artery intima and media thickness as a risk factor for myocardial infarction and stroke in older adults. Cardiovascular Health Study Collaborative Research Group. N Engl J Med 1999; 340: 14-22.

3. Johnsen SH, Mathiesen EB, Joakimsen O, Stensland E, Wilsgaard T, Løchen ML, et al: Carotid atherosclerosis is a stronger predictor of myocardial infarction in women than in men: a 6-year follow-up study of 6226 per-sons: the Tromsø Study. Stroke 2007; 38: 2873-2880.

4. Hollander M, Bots ML, Del Sol AI, Koudstaal PJ, Witteman JC, Grobbee DE, et al: Carotid plaques increase the risk of stroke and sub-types of cerebral infarction in asymptomatic elderly: the Rotterdam study. Circulation 2002; 105: 2872-2877.

5. Nambi V, Chambless L, Folsom AR, He M, Hu Y, Mosley T, et al: Carotid intima-media thickness and presence or absence of plaque improves prediction of coronary heart disease risk: the ARIC (Atherosclerosis Risk In Communities) study. J Am Coll Cardiol 2010; 55: 1600-1607.

6. Benedetto FA, Mallamaci F, Tripepi G, Zoccali C: Prognostic value of ultrasonographic measurement of carotid intima media thick-ness in dialysis patients. J Am Soc Nephrol 2001; 12: 2458-2464.

7. Nishizawa Y, Shoji T, Maekawa K, Nagasue K, Okuno S, Kim M, et al: Intima-media thick-ness of carotid artery predicts cardiovascular mortality in hemodialysis patients. Am J Kidney Dis 2003; 41(3 suppl 1):S76-S79.

8. Maeda S, Sawayama Y, Furusyo N, Shigemat-su M, Hayashi J: The association between fatal vascular events and risk factors for carotid atherosclerosis in patients on maintenance hemodialysis: Plaque number of dialytic atherosclerosis study. Atherosclerosis 2009; 204: 549-555.

9. Krediet RT, Balafa O: Cardiovascular risk in the peritoneal dialysis patient. Nat Rev Nephrol 2010; 6: 451-460.

10. Vonesh EF, Snyder JJ, Foley RN, Collins AJ: Mortality studies comparing peritoneal dialy-sis and hemodialysis: what do they tell us? Kidney Int Suppl 2006; 70:S3-S11.

11. Yilmaz FM, Akay H, Duranay M, Yilmaz G, Oztekin PS, Kosar U, et al: Carotid atherosclerosis and cardiovascular risk factors in hemodialysis and peritoneal dialysis patients. Clin Biochem 2007; 40: 1361-1366.

12. Mutluay R, Degertekin CK, Poyraz F, Yılmaz MI, Yücel C, Turfan M, et al: Dialysis type may predict carotid intima media thickness and plaque presence in end-stage renal disease patients. Adv Ther 2012; 29: 370-382.

13. Shi Z, Zhu M, Guan J, Chen J, He Q, Zhang X, et al: Dialysis methods may affect carotid intima-media thickness in Chinese end-stage renal disease patients. Ren Fail 2012; 34: 1206-1211.

14. Junyent M, Martínez M, Borràs M, Coll B, Valdivielso JM, Vidal T, et al: Predicting cardiovascular disease morbidity and mortality in chronic kidney disease in Spain. The rationale and design of NEFRONA: a prospective, multicenter, observational cohort study. BMC Nephrol 2010; 11: 14.

15. Junyent M, Martínez M, Borrás M, Bertriu A, Coll B, Craver L, et al: [Usefulness of imaging techniques and novel biomarkers in the prediction of cardiovascular risk in patients with chronic kidney disease in Spain: the NEFRONA project]. Nefrologia 2010; 30: 119-126.

16. Arroyo D, Betriu A, Martínez-Alonso M, Vi-dal T, Valdivielso JM, Fernández E: Observational multicenter study to evaluate the prevalence and prognosis of subclinical atheromatosis in a Spanish chronic kidney disease cohort: baseline data from the NEFRONA study. BMC Nephrol 2014; 15: 168.

17. La Piedra C, Fernández E, González Casaus ML, González Parra E: [Different biological functions in PTH molecules. What are we measuring?] Nefrologia 2008; 28: 123-128.

18. Stein JH, Korcarz CE, Hurst RT, Lonn E, Kendall CB, Mohler ER, et al: Use of carotid ultrasound to identify subclinical vascular disease and evaluate cardiovascular disease risk: a consensus statement from the American society of echocardiography carotid intima-media thickness task force. Endorsed by the society for vascular medicine. J Am Soc Echocardiogr 2008; 21: 93-111; quiz 189-190.

19. Touboul PJ, Hennerici MG, Meairs S, Adams H, Amarenco P, Bornstein N, et al: Mannheim carotid intimamedia thickness and plaque consensus (2004-2006-2011). An update on behalf of the advisory board of the 3rd, 
4th and 5th watching the risk symposia, at the 13th, 15th and 20th European Stroke Conferences, Mannheim, Germany, 2004, Brussels, Belgium, 2006, and Hamburg, Germany, 2011. Cerebrovasc Dis 2012; 34: 290-296.

20. Gracia M, Betriu À, Martínez-Alonso M, Arroyo D, Abajo M, Fernández E, et al: Predictors of subclinical atheromatosis progression over 2 years in patients with different stages of CKD. Clin J Am Soc Nephrol 2016; 11: 287-296.

21. Benedetto FA, Tripepi G, Mallamaci F, Zoccali C: Rate of atherosclerotic plaque formation predicts cardiovascular events in ESRD. J Am Soc Nephrol 2008; 19: 757-763.

22. Han SS, Ahn JM, Chin HJ, Chae DW, Oh KH, Joo KW, et al: Impact of C-reactive protein and pulse pressure evaluated at the start of peritoneal dialysis on cardiovascular events in the course of treatment with peritoneal dialysis. Perit Dial Int 2010; 30: 300-310.

23. Kassi E, Adamopoulos C, Basdra EK, Papavassiliou AG: Role of vitamin D in atherosclerosis. Circulation 2013; 128: 2517-2531.

24. Kunadian V, Ford GA, Bawamia B, Qiu W, Manson JE: Vitamin D deficiency and coronary artery disease: a review of the evidence. Am Heart J 2014; 167: 283-291.

25. de Boer IH, Kestenbaum B, Shoben AB, Mi-chos ED, Sarnak MJ, Siscovick DS: 25-hy-droxyvitamin D levels inversely associate with risk for developing coronary artery calcification. J Am Soc Nephrol 2009; 15: 18051812.

26. Ravani P, Malberti F, Tripepi G, Pecchini P, Cutrupi S, Pizzini P, et al: Vitamin D levels and patient outcome in chronic kidney dis-ease. Kidney Int 2009; 75: 88-95.

27. Drechsler C, Verduijn M, Pilz S, Dekker FW, Krediet RT, Ritz E, et al: Vitamin D status and clinical outcomes in incident dialysis patients: results from the NECOSAD study. Nephrol Dial Transplant 2011; 26: 1024-1032.

28. Duranton F, Rodriguez-Ortiz ME, Duny Y, Rodriguez M, Daurès JP, Argilés A: Vitamin D treatment and mortality in chronic kidney disease: a systematic review and meta-analysis. Am J Nephrol 2013; 37: 239-248.

29. Wang AY, Lam CW, Sanderson JE, Wang M, Chan IH, Lui SF, et al: Serum 25-hydroxyvi-tamin D status and cardiovascular outcomes in chronic peritoneal dialysis patients: a 3-y prospective cohort study. Am J Clin Nutr 2008; 87: 1631-1638.

30. Gracia-Iguacel C, Gallar P, Qureshi AR, Orte-ga O, Mon C, Ortiz M, et al: Vitamin D deficiency in dialysis patients: effect of dialysis modality and implications on outcome. J Ren Nutr 2010; 20: 359-367.

31. Schnatz PF, Manson JE: Vitamin D and cardiovascular disease: an appraisal of the evidence. Clin Chem 2014; 60: 600-609.

32. Kandula P, Dobre M, Schold JD, Schreiber MJ Jr, Mehrotra R, Navaneethan SD: Vitamin D supplementation in chronic kidney disease: a systematic review and meta-analysis of observational studies and randomized controlled trials. Clin J Am Soc Nephrol 2011; 6: 50-62.

33. Pilz S, Iodice S, Zittermann A, Grant WB, Gandini S: Vitamin D status and mortality risk in CKD: a metaanalysis of prospective studies. Am J Kidney Dis 2011; 58: 374-382.

34. Kerschbaum J, Vychytil A, Lhotta K, Prischl FC, Wiesholzer M, Machhold-Fabrizii V, et al: Treatment with oral active vitamin $\mathrm{D}$ is associated with decreased risk of peritonitis and improved survival in patients on peritoneal dialysis. PLos One 2013; 8:e67836.

35. Zheng Z, Shi H, Jia J, Li D, Lin S: Vitamin D supplementation and mortality risk in chronic kidney disease: a meta-analysis of 20 observational studies. BMC Nephrol 2013; 14: 199.

36. Miskulin DC, Majchrzak K, Tighiouart H, Muther RS, Kapoian T, Johnson DS, et al: Ergocalciferol supplementation in hemodialysis patients with vitamin D deficiency: a randomized clinical trial. J Am Soc Nephrol 2016; 27: 1801-1810.

37. Thadhani R, Appelbaum E, Pritchett Y, Chang Y, Wenger J, Tamez H, et al: Vitamin D therapy and cardiac structure and function in patients with chronic kidney disease: the PRIMO randomized controlled trial. JAMA 2012; 307: 674-684.

38. Verhave G, Siegert CE: Role of vitamin D in cardiovascular disease. Neth J Med 2010; 68: 113-118.

39. Perl J, Huckvale K, Chellar M, John B, Davies SJ: Peritoneal protein clearance and not peritoneal membrane transport status predicts survival in a contemporary cohort of peritoneal dialysis patients. Clin J Am Soc Nephrol 2009; 4: 1201-1206.

40. Pérez-Fontán M, Rodríguez-Carmona A, Barreda D, López-Muñiz A, Blanco-Castro N, García-Falcón T: Peritoneal protein transport during the baseline peritoneal equilibration test is an accurate predictor of the outcome of peritoneal dialysis patients. Nephron Clin Pract 2010; 116: 104-113.

41. Joffe P, Heaf JG: Vitamin D and vitamin-D-binding protein kinetics in patients treated with continuous ambulatory peritoneal dialysis (CAPD). Perit Dial Int 1989; 9: 281-284.

42. Prytula A, Wells D, McLean T, Balona F, Gullett A, Knott C, et al: Urinary and dialysate losses of vitamin Dbinding protein in children on chronic peritoneal dialysis. Pediatr nephrol 2012; 27: 643-649.

43. Lee JE, Oh KH, Choi KH, Kim SB, Kim YS, Do JY, et al: Statin therapy is associated with improved survival in incident peritoneal dialysis patients: propensity-matched comparison. Nephrol Dial Transplant 2011; 26: 40904094. 